\title{
Visualización exploratoria e interactiva de modelos de reglas de asociación
}

\author{
Exploratory and interactive visualization model for association rules \\ Wilson Castillo-Rojas ${ }^{1} \quad$ Alexis Peralta $^{1} \quad$ Camilo Vargas $^{1}$ \\ Recibido 19 de diciembre de 2014, aceptado 20 de marzo de 2015 \\ Received: December 19, 2014 Accepted: March 20, 2015
}

\begin{abstract}
RESUMEN
El artículo describe una propuesta de visualización exploratoria e interactiva para modelos de minería de datos generado con la técnica regla de asociación, aplicándole la técnica Self-Organizing Map (SOM) sobre cada componente o regla, junto a vistas proporcionadas por elementos gráficos implementados para complementar esta visualización. Esto busca establecer esquemas de visualización, que soporten la exploración visual del modelo en la etapa de ajuste del modelo de un proceso de minería de datos, y con esto responder preguntas genéricas de analistas de datos respecto del funcionamiento interno del modelo, y lograr apoyar en la comprensión del modelo generado. Este esquema de visualización propuesto se implementa por medio de un software experimental donde los analistas de datos disponen de diversos mecanismos de interacción que les permiten interactuar y explorar cada componente del modelo, con las vistas complementarias descritas con anterioridad. Finalmente, se analizan los resultados obtenidos desde un experimento controlado, llevado a cabo con un grupo de usuarios, y cuyo análisis preliminar de esta evaluación permite, por un lado, corroborar la utilidad del esquema de visualización propuesto sobre modelos de reglas de asociación, y su nivel de eficiencia en apoyar la comprensión del modelo generado.
\end{abstract}

Palabras clave: Minería de datos, minería de datos visual, visualización de modelos de minería de datos, visualización de reglas de asociación.

\section{ABSTRACT}

The paper describes a proposal for interactive visualization for exploratory data mining models generated with association rule technique, applying the Self-Organizing Map (SOM) technique on each component or rule, with a view provided by graphic elements implemented to complement this visualization. This seeks to establish visualization schemes, which support the visual exploration stage model fit of a data mining process, and with this answer generic questions regarding data analysts inner workings of the model, and achieve support in understanding generated model. This proposed scheme is implemented visualization through experimental software where data analysts have different interaction mechanisms that allow them to interact and explore each component model, with additional views described above. Finally, results from a controlled experiment, conducted with a group of users whose preliminary analysis of this evaluation allow one hand confirm the usefulness of the proposed scheme on display models of association rules, and analyzes level of efficiency in support understanding of the generated model.

Keywords: Data mining, visual data mining, data mining models visualization, association rule visualization.

\footnotetext{
1 Facultad de Ingeniería y Arquitectura. Universidad Arturo Prat. Av. Arturo Prat 2120. Iquique, Chile. E-mail: wilson.castillo@unap.cl; alexis.peralta.silva@gmail.com; vargas.medalla@gmail.com
} 


\section{INTRODUCCIÓN}

En un proceso de Minería de Datos (MD), la utilidad del modelo depende principalmente de dos factores: la capacidad del modelo para descubrir patrones interesantes, y la facilidad con la cual la estructura del modelo puede ser entendida y ajustada por usuarios/analistas de datos. Así, junto con la capacidad predictiva y descriptiva de un modelo de MD, su estructura debe ser bien entendida e interpretada por los analistas de datos. Lo anterior, debido a que la clasificación o descripción de los datos sin una explicación del modelo inducido a partir de los datos, disminuye la credibilidad de los resultados del proceso de MD [1].

En este sentido, apropiadas visualizaciones aplicadas sobre modelos de MD pueden transformar los modelos en herramientas comprensibles que convierten datos en conocimiento. Así, visualizaciones pueden transformar los modelos inducidos en cajas transparentes, tal que usuarios y analistas de datos puedan fácilmente comprender el trabajo interno que realiza el modelo, esto es, como el modelo transforma datos en patrones [2-3].

Bajo este contexto, este trabajo propone un esquema de visualización exploratoria e interactiva para modelos generados con la técnica Reglas de Asociación (RA), combinándola con la técnica SelfOrganizing Map (SOM) y aplicando visualizaciones sobre las distintas reglas o componentes del modelo. Esto busca responder preguntas genéricas de analistas de datos respecto al funcionamiento interno del modelo.

Esta propuesta se basa en un enfoque de Visualización Aumentada de Modelos para Minería de Datos, denominado VAM-MD) [4], y que plantea un modelo de percepción visual e interacción del usuario/analista de datos, centrado en la etapa de ajuste o afinamiento del modelo generado en el contexto de un proceso de MD, y establece la conveniencia de explorar el modelo original aplicándole visualizaciones con la combinación y aplicación de una segunda técnica de MD ad-hoc del tipo descriptiva, junto a un conjunto de artefactos gráficos, todos considerados "aumentadores visuales", y diversos mecanismos de interacción disponibles. Con esto se obtiene un "modelo aumentado visualmente", que permite entender su funcionamiento interno y así aportar, por un lado, a una mejor comprensión del modelo obtenido, y por otro, lograr una mayor credibilidad del modelo.

Este trabajo contempla la implementación de un esquema de visualización para modelos de RA, basado en el enfoque VAM-MD, sobre un software prototipo que permite generar un modelo de RA, sobre un conjunto de datos apropiados y previamente preparados. Luego el usuario puede seleccionar cada una de las reglas del modelo RA, y aplicar sobre estas reglas la técnica SOM, así como un conjunto de elementos visuales o artefactos gráficos implementados adecuadamente. La herramienta experimental también dispone de mecanismos de interacción que permite al usuario navegar y explorar el modelo de RA por todas sus componentes.

Finalmente, se presenta una evaluación subjetiva del software prototipo a través del desarrollo de un experimento controlado, que consiste en la encuesta a un grupo de usuarios que utilizaron esta herramienta prototipo junto a otro software de MD que no tiene implementado el enfoque VAMMD, comparándolos desde el punto de vista del entendimiento sobre el modelo logrado a través del uso de visualizaciones. Los participantes realizaron una tarea de MD diseñada para éste propósito, y proporcionaron información respecto a su desempeño, usabilidad, manejo de visualizaciones y apoyo en la comprensión del modelo MD, lo que permitió validar los resultados y contribuciones científicas de este trabajo de investigación.

\section{VISUALIZACIÓN DE REGLAS DE ASOCIACIÓN}

Las RA representan las relaciones entre varias variables unas con otras, es decir, se debe considerar que una RA es una implicación de la forma $X \rightarrow Y$, donde $X$ es un conjunto de ítems llamados antecedentes, e $Y$ es el ítem consecuente. Al menos cinco parámetros deben ser considerados en visualización de las RA: el conjunto de ítems antecedentes, ítems consecuentes, las asociaciones entre antecedentes y consecuentes, el soporte de las reglas, y la confianza de las reglas [5].

La investigación sobre visualización de RA se puede categorizar en tres grupos principales basados en: tablas, matrices, y grafos. Las técnicas basadas 
en tablas son el enfoque más común y tradicional para representar RA. Las columnas de una tabla representan generalmente los ítems, antecedentes, consecuentes, el soporte y la confianza de las RA, y cada fila representa una RA. Algunos ejemplos de técnicas basadas en tablas se pueden encontrar en los sistemas comerciales SAS Enterprise Miner y DBMiner [6].

Las técnicas basadas en matrices como las implementadas en MineSet [7] (matriz 2D), en INFOVIS [8] (matriz 3D) donde la grilla representa los antecedentes y los consecuentes son presentados en una grilla cuadrada basada en ejes de coordenadas.

El último grupo son las técnicas basadas en grafos que utilizan nodos para representar los ítems, y los bordes para representar las asociaciones de los ítems en las reglas. Algunos de estos trabajos han propuesto varios tipos de representaciones conocidas para estudiar un gran conjunto de datos, como árboles hiperbólicos [9], paredes de perspectiva [10], vistas de ojos de pez (Fish-Eyes View - FEV) [11] o superposición de vistas transparentes [12]. De estas, la vista de ojos de pez muestra el punto de interés detalladamente y la descripción del despliegue en la misma representación. Un primer acercamiento utilizando FEV en un método de visualización para RA fue propuesto en [13], en este trabajo, los autores explotaron una mezcla de representaciones para desplegar un conjunto de reglas. Actualmente varias herramientas implementan FEV como AISEE [14], INFOVIS [8] y LARM [15].

Hay otras técnicas, no especificadas en esta categorización, estas son: Gráficos de mosaicos interactivos [16], que aplica mosaicos para representar las relaciones entre ítems en cada RA desde una tabla de contingencia en vez de visualizar sus resultados de MD, CrystalClear [17] que utiliza técnica integrada por una grilla con árbol basado en el despliegue para ver el número de ítems y las listas de antecedentes/consecuentes, y una técnica propuesta por Zhao y Liu [18] que utiliza una línea para representar cada RA, el eje $x$ representa datos del tiempo, y el eje $y$ representa el valor de soporte o confianza, su visualización utiliza una técnica similar a la técnica de coordenadas paralelas [19], y finalmente una herramienta visual integrada de DM en [20]. Aunque estas tres técnicas están bien implementadas en algunos sistemas comerciales de visualización, son capaces de desplegar RA minadas, pero las visualizan todas de una sola vez en una misma pantalla, presentando demasiada información, lo que suele también generar distorsión en la interfaz, haciendo difícil su análisis para los usuarios.

En resumen, si bien es cierto que estos esfuerzos por mejorar las visualizaciones de las RA logran desplegar reglas, complementándolas con gráficos que permiten observar detalladamente cada regla, sin embargo no se logró encontrar visualizaciones que permitan interactuar con cada regla, y visualizar cómo los datos en cada regla se distribuyen espacialmente.

Otro aspecto considerado respecto de la mayoría de las herramientas de MD revisadas, es que por lo general proporcionan solo visualización de la estructura global del modelo de RA con el conjunto de reglas generadas que conforman este modelo, acompañadas con los respectivos porcentajes de soporte y confianza de cada regla, pero con poca o nula capacidad interactiva sobre el modelo y sus componentes.

De la revisión comparativa de esquemas de visualización para técnicas de MD, que realiza Castillo en [5], entre las cuales se incluyen las RA, se concluye que:

- La mayoría de las investigaciones recomiendan utilizar una combinación de técnicas de MD con apropiadas visualizaciones, dependiendo de la tarea a realizar y las características de los datos.

- Es fundamental considerar en el diseño de nuevas visualizaciones, la incorporación de mecanismos de interacción para el usuario.

- El rol de la visualización en el proceso de MD debe ser entendido y extendido en todas sus etapas, de modo que permita explorar datos, modelos y patrones.

\section{ENFOQUE VAM-MD}

El enfoque VAM-MD propuesto por Castillo en [4] considera las características de modelos de percepción analizados de Keim [21], Yan Liu [22], y Vitiello \& Kalawsky [23], recoge los aspectos más relevantes de cada uno, particularmente en lo que concierne a la integración de visualización en 
la etapa de ajuste o afinamiento y evaluación de modelos de MD.

Este enfoque aporta el concepto de "Visualización Aumentada" para modelos de MD, y propone que debido a una técnica de MD para visualizar, denominada Técnica Primaria MD (TP-MD), permitir al usuario incorporar en esta visualización diferentes elementos visuales ad hoc al tipo de modelo, y dominio de los datos, a su vez aplicar otra técnica de MD, denominada Técnica Secundaria MD (TS$\mathrm{MD}$ ), como aumentador visual que permita explorar la TP-MD. La técnica TS-MD a seleccionar debe cumplir tres requisitos: ser una técnica descriptiva, apropiada al dominio de los datos sobre con los que está trabajando la TP-MD, y aportar información complementaria a la entregada por el modelo generado por la TP-MD [4].

De modo adicional, el enfoque VAM-MD plantea la necesidad de disponer de un conjunto apropiado de mecanismos de interacción para el usuario. Todo este conjunto de visualizaciones aplicadas apuntan a lograr aumentar el análisis visual del modelo en su etapa de refinamiento o ajuste. Esta combinación de técnicas de MD es planteada por diversos autores, como mecanismos para poder lograr un mejor entendimiento, no solamente de los datos, sino también sobre los modelos generados.

La arquitectura del enfoque VAM-MD está representada en términos muy generales en la Figura 1, junto con las acciones e interacciones que el usuario dispone. La entrada en este enfoque lo constituye el conjunto de datos preparados, luego el conjunto de técnicas MD (que según sea el caso

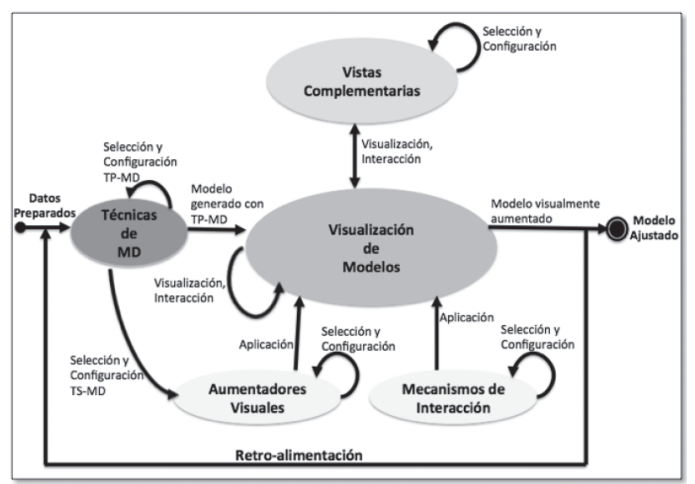

Figura 1. Arquitectura general del esquema VAM-MD [4]. serán TP o TS), un conjunto de aumentadores visuales, entre los que se disponen artefactos gráficos tradicionales y modernos (técnicas MD seleccionadas para actuar como explorador visual de otra técnica), y en la parte de abajo un conjunto de acciones que representan la disposición de diferentes mecanismos de interacción para el usuario.

En el centro del esquema se presenta la componente principal que permite visualizar el modelo de MD en la etapa de refinamiento o ajuste. Finalmente, la salida del esquema lo representa el conjunto de vistas complementarias o modelo aumentado visualmente con estas vistas.

\section{ESQUEMA DE VISUALIZACIÓN PARA MODELOS DE RA}

Particularmente para el caso de la técnica RA, la mayoría de las representaciones visuales analizadas en [5] proponen una visualización estática, sin la posibilidad de que el analista de datos pueda interactuar con cada una de las reglas.

La mayoría de las herramientas de visualización para MD revisadas, aunque entregan información general de la RA, no permiten combinar técnicas de MD que aporten información sobre reglas del modelo y las instancias en cada regla, como por ejemplo información de dispersión de los datos y su distribución espacial en cada regla, y pocas herramientas proveen mecanismos de interacción para que el usuario pueda navegar, seleccionar y explorar cada componente.

La utilización de la técnica SOM como aumentador visual aplicado a RA cumple un doble propósito: obtener la distribución espacial del subconjunto de datos asociados a cada regla, y la visualización de esta partición mediante un mapa. Considerando que las RA permiten establecer un conjunto de reglas que asocian atributos según el factor de ocurrencia simultánea, y que cada regla recopila las instancias que cumplen con estas asociaciones, sin embargo, no permiten visualizar la distribución espacial de estas instancias en cada regla, lo que sí permite proveer la técnica SOM. Además, la técnica SOM es apropiada al dominio de los datos que maneja la RA, y permite describir la distribución de estos datos en cada uno de sus componentes. 


\section{Prototipo Experimental}

El software prototipo desarrollado tiene como alcance para este análisis experimental la implementación del esquema VAM-MD, para una técnica MD del tipo jerárquico, particularmente para este estudio la técnica RA en combinación con la técnica SOM como TS-MD o aumentador visual para su exploración y análisis.

Además, incorpora el set de elementos visuales compuesto por: tabla de datos, gráficos circular global y por regla, gráfico de puntos, gráfico de fuerza y diagrama de coordenadas paralelas.

También, dispone de los mecanismos de interacción: zoom (in/out), selección de reglas, y ajuste de parámetros.

En la Figura 2 se puede observar la interfaz principal del prototipo experimental, donde se visualiza un modelo de RA en la parte central, junto a vistas complementarias y elementos visuales en el lado derecho. En esta herramienta se implementan todas las componentes que la arquitectura del esquema VAM-MD plantea en la Figura 1:

a) Selección, configuración de parámetros de la TP-MD, y generación del modelo de minería de datos: esta sección cuenta con dos pestañas, la primera "Datos", por donde parte la herramienta permitiendo al usuario seleccionar el conjunto de datos a ser analizados, y luego mostrar algunas características de estos datos y sus atributos, como por ejemplo; número de instancias y atributos, tipo y cantidad de atributos, etcétera.

En la segunda pestaña “T. Primaria MD”, el usuario puede seleccionar la Técnica Primaria de Minería de Datos (TP-MD), la que es explorada con otra técnica de MD combinada con elementos visuales. También, el usuario puede configurar los parámetros iniciales de esta técnica, para luego ser ejecutada generando el modelo de MD.

b) Área de visualización para TP-MD: es el área de trabajo principal donde se presenta la vista general de la TP-MD seleccionada, donde el usuario puede interactuar (navegar, explorar y seleccionar) con cada elemento o regla. Cuenta con dos pestañas, una llamada "Datos" donde se muestran los atributos y los datos originales del conjunto de datos a ser analizados. La segunda pestaña "Técnica Primaria MD" muestra la técnica seleccionada que para este caso es un RA.

c) Técnica secundaria de MD como aumentador visual: se muestra en esta área una vista minimizada de la TS-MD, que el usuario selecciona y aplica

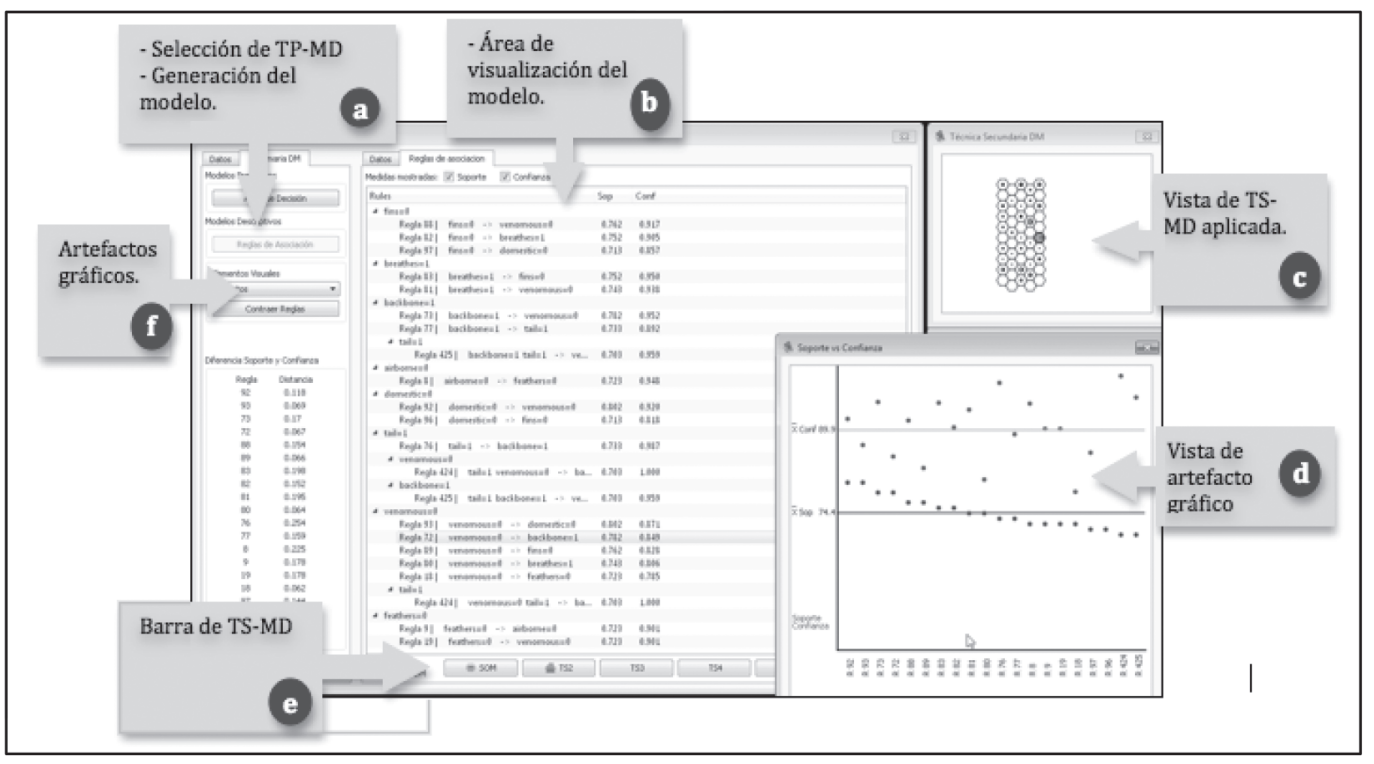

Figura 2. Interfaz principal del prototipo experimental. 
sobre la TP-MD. También el usuario en esta área puede maximizar la vista, permitiendo modificar sus parámetros de configuración. Para este trabajo se considera la técnica SOM como aumentador visual o TS-MD.

d) Elemento visual; en esta zona se despliegan los elementos visuales según sean seleccionados desde la sección f), por ejemplo, en la Figura 2 se puede observar un gráfico de puntos que grafica todas las reglas respecto del promedio de soporte y confianza general. Una de la características que tiene este cuadro, es que también cuenta con el mecanismo de interacción zoom (in/out) ya que al pasar por encima con el mouse y salir de este gráfico, su tamaño aumenta o vuelve a su estado original.

e) Barra de aumentadores visuales: se ubica en la parte inferior del área de trabajo y presenta un set de aumentadores visuales o TS-MD, que el usuario puede seleccionar para aplicar sobre la TP-MD, y con esto poder explorar cada componente del modelo generado. Para este trabajo se muestra la selección del ícono de la técnica SOM.

f) Selección de elemento visual: en esta sección el usuario dispone de un conjunto apropiado de artefactos gráficos que puede seleccionar, configurar y aplicar sobre la TP-MD, y su gráfica se presenta en el cuadro d). Dentro del conjunto de elementos visuales se dispone de gráfico circular global y por cada regla, gráficos de fuerza y puntos, y diagrama de coordenadas paralelas.

Una vez que el usuario aplica la técnica SOM (TSMD) sobre una regla, el usuario puede maximizar la imagen ubicada en la sección c) de la interfaz al hacer clic con el mouse, lo que abre una ventana que presenta una vista detallada de esta técnica, y puede reconfigurar sus parámetros iniciales.

Como se observa en la Figura 3, sobre una regla seleccionada y aplicada la técnica SOM, el usuario puede visualizar la forma de distribución de las instancias en esta regla. Además, dispone de elementos de interacción para cambiar el tipo de color del fondo del mapa y el tipo de clase, el tipo de gráfico de cada ítem del mapa, elegir el tipo de clase a colorear en la representación, seleccionar, visualizar el conjunto de prueba o entrenamiento, etcétera.

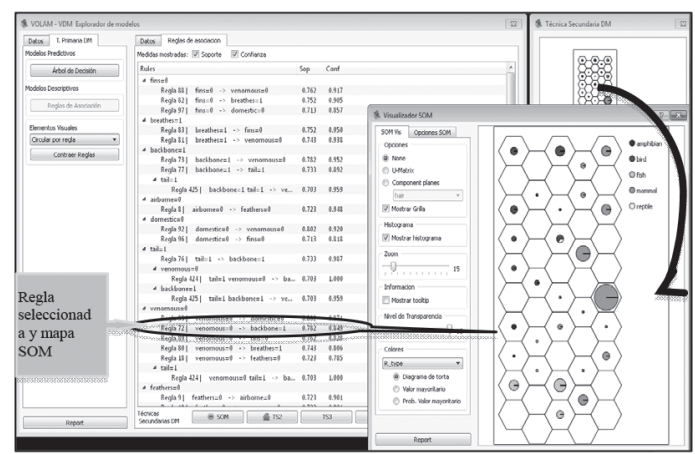

Figura 3. Técnica SOM aplicada a una RA.

\section{EVALUACIÓN Y ANÁLISIS DE RESULTADOS}

\section{Experimento controlado}

El experimento controlado contempla la evaluación comparativa y subjetiva de la visualización del modelo de RA obtenido mediante el desarrollo de una tarea de MD que deben realizar un conjunto de usuarios/analistas de datos, y cuyo objetivo es comprobar si el esquema de visualización aumentada del modelo de RA con la técnica SOM junto al conjunto de elementos visuales proporcionados por el software prototipo, permiten mejorar la comprensión del modelo, como por ejemplo observar la distribución de sus datos en cada regla, comparándolo con la visualización provista por otra herramienta de MD que no tiene este enfoque o esquema de visualización.

Este experimento se realizó con 17 personas de distintos niveles de conocimientos en procesos de MD y en el uso de herramientas de MD. Se utilizó el software WEKA (Waikato Environment for Knowledge Analysis) como herramienta de MD para contrastar el aporte del prototipo. El motivo de utilizar WEKA para la medición es porque esta herramienta no tiene implementada visualización de las RA bajo este enfoque o similar, lo que permitió comparar si aumentando visualmente las RA con SOM, mejora sustantivamente la comprensión del modelo, respecto de no tener esta visualización.

Con ambas herramientas los usuarios participantes del experimento debieron generar un modelo de RA, luego visualizar e interactuar con el modelo, para posteriormente interpretar los patrones o reglas obtenidas, utilizando todas las opciones que los dos 
softwares ofrecen. Se buscó que los participantes realicen una tarea genérica de descripción, y pudieran responder preguntas respecto del modelo, sus componentes, y relacionar el modelo con las características de los datos desde el cual el modelo fue generado.

Posteriormente, una vez realizada la tarea de MD preparada para este experimento, los usuarios debieron responder una encuesta diseñada para recoger la opinión subjetiva del grupo, respecto del desempeño de ambas herramientas en el manejo de visualización del modelo del RA generado, su usabilidad, la utilidad de los elementos visuales proporcionados, la conveniencia de combinar y aplicar la técnica SOM a modelos de RA para lograr un modelo visualmente aumentado, y la eficiencia en la comprensión del modelo generado.

\section{Análisis de resultados}

En general, la experiencia de desarrollar una tarea de MD para generar un modelo RA, utilizando el software prototipo tuvo una gran aceptación, desde el punto de vista de la usabilidad y desempeño, manifestando el $94,12 \%$ que esta herramienta ofrece visualizaciones que describen mejor el modelo, comparativamente con una herramienta que no dispone esta combinación de visualizaciones.

Los usuarios en su mayoría manifestaron que tanto la combinación de la técnica SOM aplicada sobre el modelo de RA, y el uso de los elementos gráficos sobre los datos de las reglas, les permitieron mejorar la comprensión del modelo de RA generado, logrando una valoración de 54,9\% bueno y $33,33 \%$ muy bueno, lo que se puede observar del gráfico en la Figura 4.

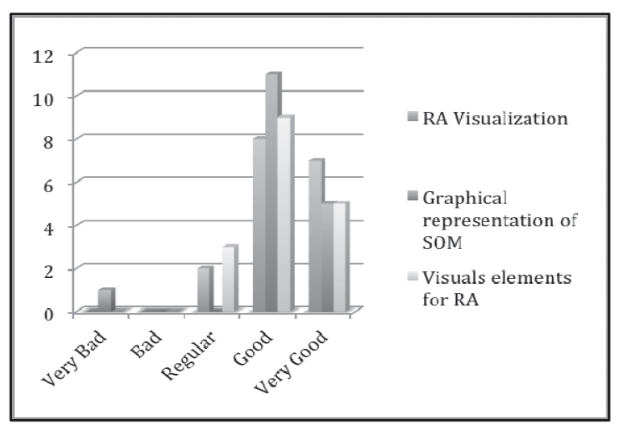

Figura 4. Nivel de aceptación de visualizaciones disponibles para describir el modelo RA.
El gráfico de la Figura 5 detalla el nivel de utilidad de cada uno de los elementos visuales o gráficos, que el usuario aplicó sobre los datos de las reglas en el modelo de RA, y que lograron en forma agrupada una valoración alta $37,97 \%$ y muy alta con un $32,91 \%$ respecto del aporte como vista complementaria para describir el modelo de RA. Se puede observar que el gráfico de puntos fue el mejor evaluado, mientras que los gráficos circulares global, y por regla fueron los que obtuvieron la menor calificación.

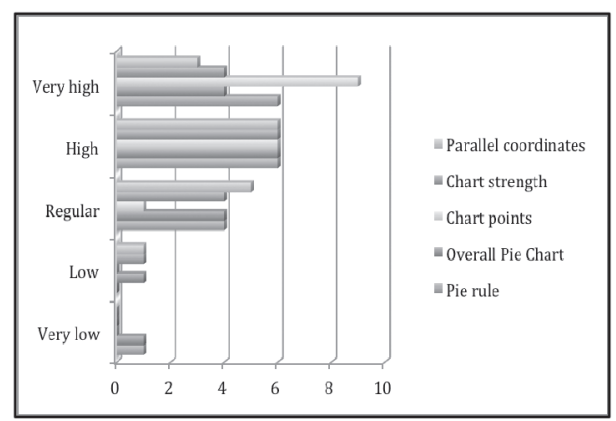

Figura 5. Detalle del nivel de valoración de los elementos visuales.

Finalmente en el gráfico de la Figura 6, los usuarios ante la consulta: si la visualización aumentada del modelo de RA, aplicándole la técnica SOM en cada una de sus reglas, les provee una visión amplificada de los datos y su distribución espacial, permitiendo describir el comportamiento de los datos en cada regla, los usuarios manifiestan en su mayoría positivamente (alto $76,5 \%$ y muy alto $11,8 \%$ ) que si se logra obtener visualización aumentada del modelo de RA.

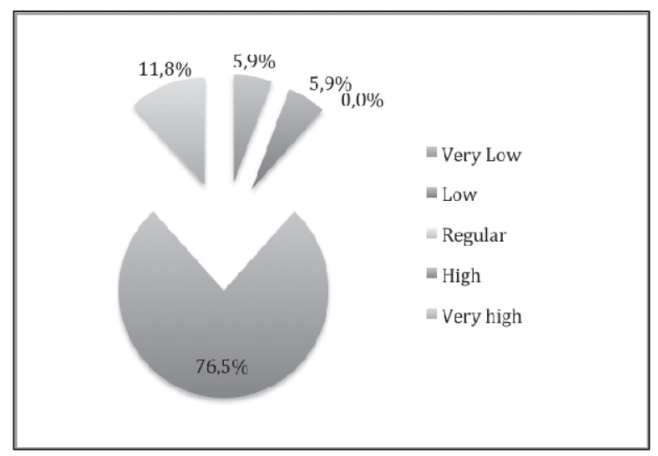

Figura 6. Capacidad de describir los datos en el modelo RA mediante el uso de la técnica SOM. 


\section{CONCLUSIONES Y TRABAJO FUTURO}

Los resultados preliminares de este trabajo permiten confirmar la idoneidad y utilidad de combinar RA con la técnica SOM, logrando obtener una visualización aumentada del modelo de RA, y la distribución espacial sobre los datos de cada regla. Las vistas complementarias provistas por los elementos visuales o gráficos, también logran un nivel de aceptación alto destacando por sobre todo el gráfico de puntos. Como, contraparte los usuarios manifestaron que algunos gráficos no son un aporte visual, específicamente los gráficos circulares global y por regla.

Se puede deducir con los resultados de esta experimentación, que la aplicación de la técnica SOM sobre un modelo de RA, puede mejorar la comprensión de su funcionamiento interno. También que los elementos visuales o gráficos proporcionados en el software prototipo, aplicado sobre los datos de cada regla del modelo, apoyan el análisis y exploración del modelo de RA generado.

Como trabajo futuro se están evaluando otras técnicas de MD, del tipo descriptiva, que puedan proporcionar vistas complementarias a las que entrega la técnica de SOM para aumentar, entre estas se considera preliminarmente técnica de clustering. También se contempla implementar nuevos artefactos gráficos que puedan ser más útiles en la exploración de los datos para las reglas del modelo de RA.

\section{REFERENCIAS}

[1] D.A. Keim. "Visual Techniques for Exploring Databases". Tutorial Notes in the Third International Conference on Knowledge Discovery and Data Mining. Newport Beach, CA, EE.UU. August, 1997.

[2] C.J. Meneses and G.G. Grinstein. "Visualization for Enhancing the Data Mining Process". In Proceedings of the Data Mining and Knowledge Discovery: Theory, Tools, and Technology III Conference. Orlando, FL, EE.UU, pp. 16-20. April, 2001.

[3] K. Thearling, B. Becker, D. DeCoste, B. Mawby, M. Pilote and D. Sommerfield. "Visualizing Data Mining Models". In Proceedings of the Integration of Data
Mining and Data Visualization Workshop. Springer-Verlag. 1998.

[4] W. Castillo-Rojas, C.J. Meneses and F. Medina. "Augmented Decision Tree Models Using SOM". Article presented in 6th Latin American Conference on Human Computer Interaction-CLIHC 2013, Carrillo, Costa Rica. Proceedings, pp. 148-155. Springer LNCS 8278. ISBN: 978-3-319-03067-8. November, 2013.

[5] W. Castillo-Rojas and C.J. Meneses. "Comparative Review of Schemes of Multidimensional Visualization for Data Mining Techniques". III Congreso Internacional de Computación e Informática del Norte de Chile. Arica, Chile. 8-10 de agosto de 2012.

[6] J.Han and M. Kamber. "Data Mining Concepts and Techniques". Morgan Kaufmann. 2001.

[7] C. Brunk, J. Kelly and R. Kohavi. "MineSet: An Integrated System for Data Mining". Proc. of Third Intel: Knowledge Discovery and Data Mining, pp. 135-138. 1997.

[8] P.C. Wong, P. Whitney and J. Thomas. "Visualizing association rules for text mining". In INFOVIS, pp. 120-123. 1999.

[9] J. Lamping, R. Rao and P. Pirolli. "A focus+context technique based on hyperbolic geometry for visualizing large hierarchies". In Proceedings of the ACM conference on Human Factors in Computing Systems (CHI'95). ACM Press. Denver, Colorado, USA, pp. 401-408. 1995.

[10] J. Mackinlay, G.G. Robertson and S.K. Card. "The perspective wall: Detail and context smoothly integrated". In Proceedings of the ACM conference on Human Factors in Computing Systems (CHI'91). ACM Press, pp. 173-179. 1991.

[11] G.W. Furnas. "A fisheyes follow-up: Further reflections on focus+context". In Proceedings of the ACM conference on Human Factors in Computing Systems (CHI'06). ACM Press. Montreal, Canada, pp. 999-1008. 2006.

[12] B.L. Harrison and K.J. Vicente. "An experimental evaluation of transparent menu usage". In Proceedings of the ACM conference on Human factors in computing systems (CHI'96), pp. 391-398. ACM Press. USA. 1996. 
[13] O. Couturier, J. Rouillard and V. Chevrin. "An interactive approach to display large sets of association rules". In Proceedings of the 12th International Conference on Human-Computer Interaction (HCI' 07). Beijing, China. 2007.

[14] -AbsInt. "aiSee-Graph Visualization". URL: http://www.aisee.com. Date of visit: December 17, 2012.

[15] O. Couturier, E. Mephu Nguifo and B. Noiret. "A formal approach to occlusion and optimization in association rules Visualization". In Proceedings of International Symposium of Visual Data Mining (VDM) of IEEE 9th International Conference on Information Visualization (IV@VDM' 05). London, UK. July, 2005.

[16] H. Hofmann, A.P. Siebes and A.F. Wilhelm. "Visualizing association rules with interactive mosaic plots". In Proceedings of the sixth ACM SIGKDD international conference on Knowledge discovery and data mining. ACM Press, pp. 227-235. 2000.

[17] K.H. Ong, K.L. Ong and E.P. Lim. "Crystalclear: Active visualization of association rules". In International Workshop on Active Mining, in conjunction with IEEE International Conference on Data Mining. 2002.

[18] K. Zhao and B. Liu. "Visual analysis of the behavior of discovered rules". In: Workshop Notes in ACM SIGKDD Workshop on Visual Data Mining. 2001.
[19] A. Inselberg and B. Dimsdale. "Parallel coordinates for visualizing multidimensional geometry". Computer Graphics. Proceedings of CG International, pp. 25-44. 1987.

[20] K. Techapichetvanich and A. Datta. "Visual mining of market basket association rules". In Computational Science and Its Applications ICCSA International Conference. Proceedings, Part IV. Vol. 3046 of LNCS, pp. 479-488. Assisi, Italy. May 14-17, 2004.

[21] D. Keim, J. Kohlhammer, E. Geoffrey and F. Mansmann. "Mastering the Information Age Solving Problems with Visual Analytics". Printed in Germany, Druckhaus Thomas Müntzer GmbH, Bad Langensalza. Theoretical Issues in Ergonomics Science. Vol. 8, Issue 1, pp. 63-92. ISBN: 978-3905673-77-7. January-February, 2007.

[22] Y. Liu and G. Salvendy. "Visualization support to better comprehend and improve decision tree classification modelling process: a survey and appraisal". Theoretical Issues in Ergonomics Science. ISSN: 1463-922X (print) / ISSN: 1464-536X. Taylor \& Francis. URL: http://www.tandf.co.uk/ journals. DOI: 10.1080/1463922050028 4371. 2007.

[23] P.F. Vitiello and R.S. Kalawsky. "Visual Analytics: A Sensemaking Framework for Systems Thinking in Systems Engineering". Systems Conference (SysCon). IEEE International Conference Publications. 978-1-4673-0750-5/12/\$31.00. ISBN: 9781-4673-0748-2, 2012. 